\title{
Narrow-band photometry of the eclipsing WN7+O binary WR 22
}

\author{
Pierre Royer $^{1}$, Gregor Rauw ${ }^{1}$, Jean Manfroid ${ }^{1}$, Eric Gosset $^{1}$, and \\ Jean-Marie Vreux \\ Institut d'Astrophysique et de Géophysique, Université de Liège, \\ 5, Avenue de Cointe, B-4000 Liège, Belgium
}

\begin{abstract}
In the present paper, we discuss photometric observations of the February 1996 eclipse of the very massive WR+O binary WR 22. Our data were obtained with a set of narrow-band filters, specially designed for the study of WR stars.
\end{abstract}

\section{Introduction}

The WN7+abs+O binary WR $22\left(P_{\text {orb }}=80.3 \mathrm{~d}\right)$ displays a shallow eclipse around its periastron passage, when the WR star is in front of its companion (Balona et al. 1989; Gosset et al. 1991). Such an atmospheric eclipse results from the phase-dependent opacity along our sightline towards the $\mathrm{O}$ star and is a common feature among $\mathrm{WR}+\mathrm{O}$ binaries with orbital periods shorter than 30 days (Lamontagne et al. 1996).

In the case of WR 22 , as a result of the combination of the inclination of the orbit, of its eccentricity $(e \simeq 0.56)$, and of the argument of the periastron $\left(\omega \simeq 272^{\circ}\right)$, only the secondary eclipse near periastron is present, whereas no occultation is observed near apastron (Gosset et al. 1991; Rauw et al. 1996).

\section{Narrow-band photometry of WR 22}

Photometric observations, through specially designed narrow-band filters, of the secondary eclipse of WR 22 were acquired during five nights in February 1996 (JD 2450125-129) at the Bochum $0.6 \mathrm{~m}$ telescope (La Silla, Chile) equipped with the DLR CCD camera using a Tektronix $1024 \times 1024$ chip (pixel size $23 \mu \mathrm{m}$ ). For a detailed description of the characteristics and performances of our set of narrowband filters, see Royer et al. (1998). The data were reduced using the algorithm described by Manfroid (1993).

Given the incomplete phase-coverage of the eclipse by the narrow-band observations, we have fitted the data with the mean light-curve, as derived from a more extensive data set obtained in the Strömgren $y$ filter, keeping the depth of the eclipse as the only free parameter (see Fig. 1). The Strömgren data set will be discussed and analysed in a forthcoming paper (Rauw et al. in preparation).

\footnotetext{
${ }^{1}$ At the Fonds National de la Recherche Scientifique, Belgium
} 


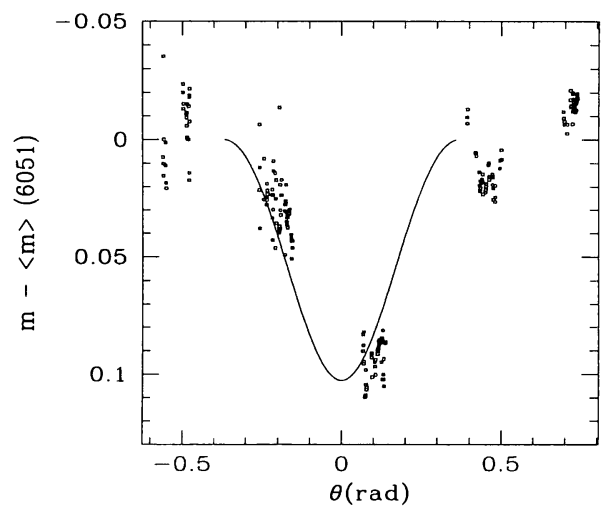

Figure 1. Light-curve of WR 22 as observed with a filter centered on $6051 \AA$. Differential magnitudes are shown as a function of $\theta=\psi-\frac{3 \pi}{2}+\omega$ where $\psi$ is the true anomaly and $\omega$ the argument of the periastron (orbital elements from Rauw et al. 1996).

As expected, the eclipse as observed with the filter centered on the He II $\lambda 4686$ emission line is shallower than with the continuum filters (centered on $\lambda 5057$ and $\lambda 6051)$. The fitted depths are $\Delta \mathrm{m}(\lambda 4686)=0.062, \Delta \mathrm{m}(\lambda 5057)=0.112$ and $\Delta \mathrm{m}(\lambda 6051)=0.102$. The depths of the eclipse in the continuum filters provide upper limits on the luminosity ratio $\mathrm{L}_{\mathrm{WR}} / \mathrm{L}_{\mathrm{O}}$ of $9.2(\lambda 5057)$ and 10.1 $(\lambda 6051)$. We have performed numerical simulations to quantify the contribution of the He II $\lambda 4686$ line to the integrated flux in the narrow filter as a function of the line's orbital Doppler shift. On the average, the emission line contributes for some $42 \%$ of the total flux and hence accounts for the depth difference of the eclipse as observed with the He II $\lambda 4686$ filter compared to the continuum filters. During the phase interval $\phi=0.97 \rightarrow 0.03$, the radial velocity of the He II $\lambda 4686$ line increases from $\sim 0$ to $\sim+120 \mathrm{~km} \mathrm{~s}^{-1}$. Due to the Doppler shift of the line within the narrow filter passband, our simulations, accordingly, yield an apparent faintening by 0.007 magnitude during this phase interval, i.e., too small an effect to be detected in our data given their accuracy and the intrinsic variability of the WN7+abs star. In contrast to most of the light-curves of eclipsing WR+O systems reported by Lamontagne et al. (1996), the light-curve of WR 22 displays a rather narrow shape without prominent extended wings, pointing towards a rather low mass-loss rate $(\log \dot{M} \leq-4.8$, Rauw 1997).

\section{References}

Balona, L.A., Egan, J., Marang, F. 1989, MNRAS 240, 103

Gosset, E., Remy, M., Manfroid, J., et al. 1991, IBVS 3571

Lamontagne, R., Moffat, A.F.J., Drissen, L., Robert, C., Matthews, J.M. 1996, AJ 112, 2227

Manfroid, J. 1993, A\&A 271, 714

Rauw, G. 1997, PhD Thesis, Université de Liège

Rauw, G., Vreux, J.-M., Gosset, E., et al. 1996, A\&A 306, 771

Royer, P., Vreux, J.-M., Manfroid, J. 1998, A\&AS 130, 407 